'Servicio de Dermatología, Hospital Clínico San Borja

Arriarán. Santiago, Chile. 2Departamento de Dermatología, Facultad de Medicina, Universidad de Chile. Santiago, Chile.

${ }^{3}$ Departamento de Medicina Interna, Campus Centro, Facultad de Medicina, Universidad de Chile. Santiago, Chile.

${ }^{4}$ Servicio de Medicina, Hospital Clínico San Borja Arriarán. Santiago, Chile. ${ }^{5}$ Servicio de Anatomía Patológica, Hospital Clínico San Borja Arriarán. Santiago, Chile.

Trabajo no recibió financiamiento. Los autores declaran no tener conflictos de interés.

Recibido el 29 de julio de 2018 , aceptado el 7 de diciembre de 2018.

Correspondencia a: Pablo Vargas Santos Dumont 999 Independencia, Santiago, Chile. pablovargas.med@gmail.com

\section{Síndrome de DRESS con compromiso pulmonar extenso. Una presentación inhabitual}

\author{
JAVIER ARELLANO ${ }^{1,2}$, PABLO VARGAS ${ }^{2}$, CLAUDIA MARTÍNEZ ${ }^{3}$, \\ MARCO CHAHUAN ${ }^{3,4}$, YAMILE CORREDOIRA $^{5}$
}

\section{Drug rash with eosinophilia and systemic symptoms or DRESS syndrome. Report of one case}

Drug rash with eosinophilia and systemic symptoms or DRESS Syndrome is a rare, serious and potentially fatal adverse drug reaction. It is characterized by widespread morbilliform and edematous skin lesions, associated with eosinophilia, lymphadenopathy and internal organ involvement and unusually associated with pulmonary symptoms. We report a 47-year-old male with DRESS syndrome, manifested with typical skin lesions and extensive pulmonary involvement, responding satisfactorily to systemic corticosteroids.

(Rev Med Chile 2019; 147: 114-118)

Key words: Drug Hypersensitivity; Drug Hypersensitivity Syndrome; Pneumonia.
L as reacciones adversas a medicamentos representan una causa importante de morbimortalidad a nivel mundial y, de acuerdo a esto, se clasifican en simples o complejas. Dentro de este último grupo, la reacción por drogas con eosinofilia y síntomas sistémicos (síndrome de DRESS, por su nombre en inglés, Drug rash with eosinophilia and systemic symptoms), es una entidad infrecuente, grave y potencialmente mortal, caracterizada por síntomas cutáneos y compromiso multisistémico ${ }^{1,2}$. Pese a que el término fue acuñado en 1996 por Bocquet et $\mathrm{ll}^{3}$, esta fue descrita varias décadas antes, en la década 1930-39, asociada a fenitoína y su novel salida al mercado ${ }^{4}$. Posteriormente, se vinculó etiológicamente a otros fármacos anticonvulsivantes aromáticos, además de antibióticos, antivirales, antidepresivos, antihipertensivos, antiinflamatorios no esteroidales, biológicos, entre otros ${ }^{1,5}$. Respecto al compromiso sistémico, puede afectar a múltiples órganos, sin ser habitual el compromiso pulmonar como manifestación principal ${ }^{6,7}$. Por esto, se presenta el caso clínico de un paciente con síndrome de DRESS, cuya principal manifestación clínica fue disnea y extenso infiltrado pulmonar difuso, asociado a penicilina benzatina y metamizol sódico.

\section{Caso clínico}

Paciente de sexo masculino, 47 años, obrero de la construcción, con tabaquismo activo (índice paquete año 34), sin otros antecedentes mórbidos relevantes. Inició tos no productiva, coriza, odinofagia y sensación febril, de 3 días de evolución, por lo que se indicó penicilina benzatina 1,2 millones de unidades y metamizol sódico $1 \mathrm{~g}$, ambos por vía intramuscular, con el diagnóstico de amigdalitis aguda. Luego de 5 días, ingresó por disnea de esfuerzo leve a moderada y lesiones cutáneas pruriginosas en cuello, tórax y abdomen, de rápida extensión. Al examen físico, destacaba: paciente afebril, taquicárdico, saturando $\mathrm{O}_{2} 92 \%$ (ambiental), a la auscultación pulmonar con 
sibilancias bilaterales y crépitos en base derecha, además de múltiples pápulas y placas urticariformes eritematosas, ligeramente descamativas y parcialmente confluentes, distribuidas con predominio en región dorsolumbar y cervical, más algunas lesiones aisladas en tórax anterior y abdomen (Figura $1 \mathrm{~A}-\mathrm{C}$ ). Se tomaron biopsias de piel, que mostraron dermatitis perivascular superficial y profunda, linfoide con eosinófilos, psoriasiforme y de interfase vacuolar, compatible con reacción adversa a fármacos.

En exámenes complementarios, destacaban: leucocitos de $32.000 \times \mathrm{xm}^{3}$, con $6,1 \%$ de eosinófi$\operatorname{los}(2.000)$ y $74,7 \%$ segmentados, proteína $C$ reactiva $20,8 \mathrm{mg} / \mathrm{dl}$. Se realizó panel de inmunofluorescencia indirecta para virus respiratorios, antígeno de superficie para virus hepatitis $B$, anticuerpos de virus hepatitis $\mathrm{C}$, VIH, VDRL y hemocultivos, resultando todos negativos. Además, se realizó reacción de polimerasa en cadena cualitativa en sangre, para virus herpes humano (VHH) 6, que resultó negativa. A los 5 días de evolución, presentó alza progresiva en transaminasas, con glutámico oxalacética (GOT) de 127 U/L, glutámico pirúvica de $436 \mathrm{U} / \mathrm{L}$, gamma glutamiltranspeptidasa (GGT) de 395 U/L y fosfatasas alcalinas de 237 U/L. Radiografía de tórax al ingreso se interpretó como neumonía multifocal. Se realizó tomografía axial computarizada de tórax con contraste, que mostró compromiso difuso intersticio-alveolar bilateral y adenopatías mediastínicas (Figura 1D). Se realizó lavado broncoalveolar, evidenciando células epiteliales escamosas, células cilíndricas ciliadas, macrófagos y polimorfonuclares, sin eosinófilos. Cultivos negativos.

Considerando la eosinofilia, el compromiso pulmonar, hepático y biopsia de piel compatible, se diagnostica síndrome de DRESS, suspendiendo antiinflamatorios no esteroidales e iniciando tratamiento con prednisona $1 \mathrm{mg} / \mathrm{kg} /$ día, evolucionan-

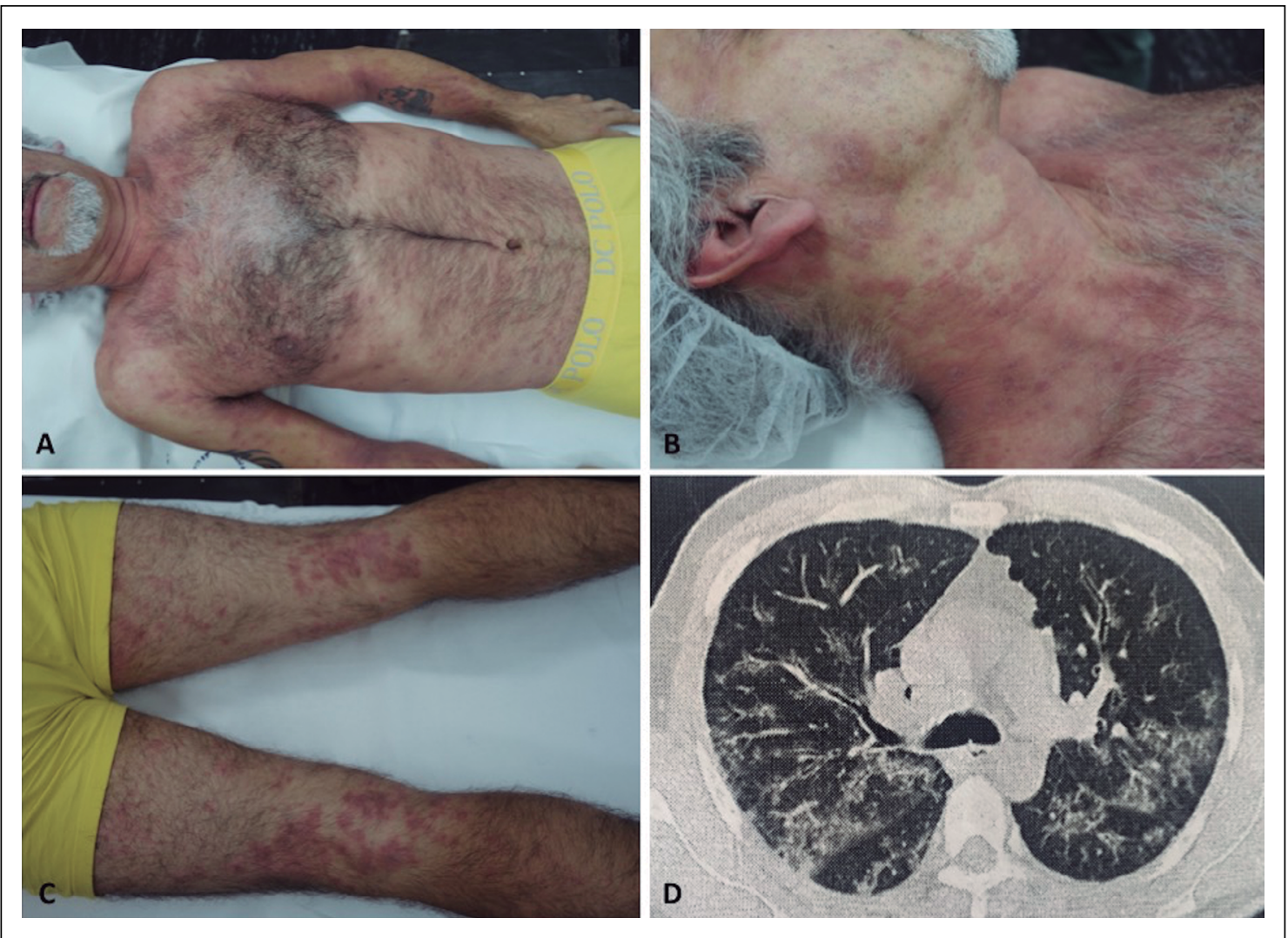

Figura 1. A-C) Pápulas y placas urticariformes eritematosas, confluentes y ligeramente descamativas. D) Infiltrado difuso intersticio-alveolar bilateral. 


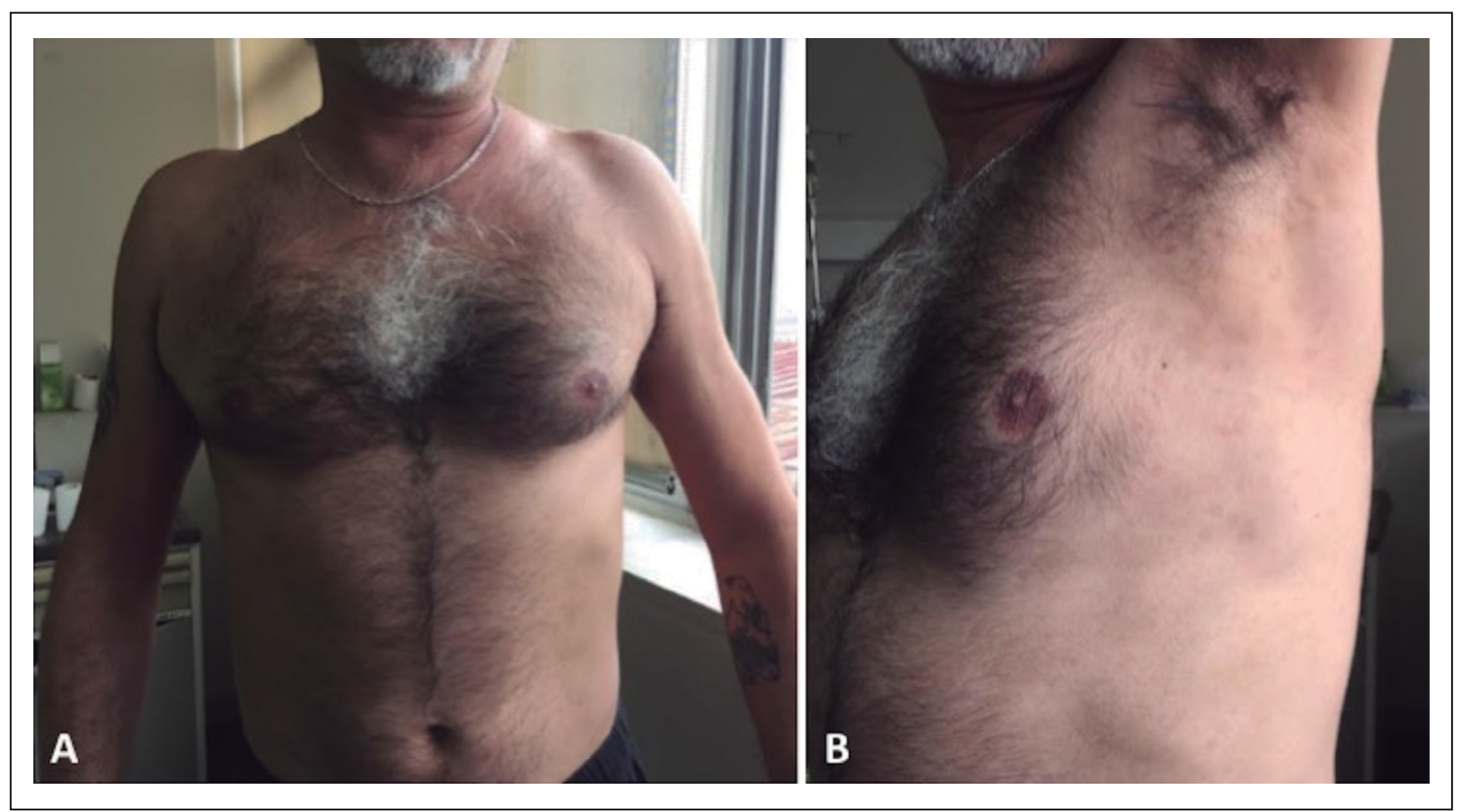

Figura 2. A-B) Resolución de lesiones cutáneas luego de tratamiento con corticoides sistémicos. Tres semanas de evolución.

do favorablemente, con desaparición de lesiones cutáneas e imágenes pulmonares en tomografía axial computarizada de tórax de control, a las 3 semanas de seguimiento (Figura 2).

\section{Discusión}

El síndrome de DRESS corresponde a una reacción adversa a fármacos grave, mediada por una respuesta de hipersensibilidad tipo IVb, asociado a drogas o sus metabolitos, con un período de latencia entre la exposición y el inicio de síntomas, clásicamente de 2 a 6 semanas $^{1,8}$, siendo descrito períodos con rangos más amplios, entre 3 y 105 días 2 . Se ha atribuido un rol etiopatogénico a un déficit enzimático en el metabolismo de fármacos en individuos predispuestos, además de un eventual rol de ciertos virus, principalmente VHH tipo 6 , que resultó negativo en nuestro paciente. También se ha relacionado a $\mathrm{VHH} 7$, citomegalovirus y virus Epstein Barr ${ }^{9}$, los que no fueron evaluados en presente caso.

Clínicamente se presenta con una fase prodrómica de fiebre y prurito, seguida clásicamente de exantema eritematoso morbiliforme, que afecta cara, tronco superior y extremidades, evolucionando a lesiones infiltradas y edematosas difusas, comprometiendo gran extensión cutánea, generando incluso eritrodermia ${ }^{2,10}$. Otras manifestaciones dermatológicas menos frecuentes son lesiones vesiculares, bulas, placas targetoides y púrpura, además de eventual compromiso oral, con erosiones mucosas, eritema faríngeo y queilitis ${ }^{1}$. En $75 \%$ de los casos cursan con linfadenopatías localizadas o generalizadas y hasta en $90 \%$ leucocitosis con eosinofilia, que puede presentarse tardíamente, luego de 1 a 2 semanas de evolución, además de linfocitosis atípica ${ }^{2,5}$. Respecto al compromiso visceral, es más frecuente a nivel hepático y renal, reportándose también afección pulmonar ${ }^{6,8}$. El compromiso pulmonar del síndrome de DRESS es inhabitual, estimándose una prevalencia entre 5 y $33 \%$, probablemente relacionado al subrreporte de casos con compromiso pulmonar menos grave $\mathrm{g}^{7,8}$. La información al respecto es escasa y está basada fundamentalmente en la publicación de casos clínicos, en los que se ha observado variadas manifestaciones, reportándose: enfermedad pulmonar difusa, derrame pleural, distrés respiratorio, hemorragia alveolar, compromiso bronquial y neumomediastino ${ }^{6,8}$. El caso clínico que presentamos tuvo compromiso alveolar con imágenes 
en vidrio esmerilado y afectación de la vía aérea periférica tipo "árbol en brote". Los síntomas clínicos más frecuentes son disnea y tos, ambos manifestados en el caso presentado. La gravedad puede ser de cuadros leves a muy graves, con riesgo vital ${ }^{8}$.

El principal fármaco involucrado en el compromiso pulmonar es la minociclina ${ }^{11}$, reportándose además antivirales ${ }^{12,13}$, terapia homeopática ${ }^{14}$, anticonvulsivantes y dapsona ${ }^{11}$, no encontrando en la literatura casos como nuestro paciente, asociado a metamizol o betalactámicos, pese a que los antibióticos representan una causa importante de síndrome de DRESS ${ }^{1}$.

En muchas ocasiones, el diagnóstico representa un desafío para los equipos de salud, dado principalmente por la heterogeneidad clínica, tanto en el compromiso cutáneo como multisistémico. En este sentido, el grupo europeo de reacciones cutáneas a drogas elaboró el RegiS$\mathrm{CAR}^{15}$, basado en una puntuación de acuerdo a los diversos hallazgos clínicos y de laboratorio, definiendo sus resultados como: no diagnóstico, caso posible, caso probable y caso definitivo. En una revisión retrospectiva de 172 casos, utilizando el RegiSCAR, se encontró un diagnóstico probable o definitivo en $88 \%{ }^{7}$, constituyendo una importante herramienta diagnóstica.

Respecto al tratamiento, este se basa fundamentalmente en la rápida suspensión del fármaco causante, además del inicio precoz de corticoterapia sistémica en dosis equivalentes a prednisona 1 $\mathrm{mg} / \mathrm{kg} /$ día, siendo recomendada la disminución progresiva en 3 a 6 meses posterior a la mejoría clínica y de laboratorio. En casos leves, pudiera considerarse solo corticoides tópicos de alta potencia. En casos graves y refractarios a corticoides, se describen alternativas como: inmunoglobulina iv, plasmaféresis, ciclosporina, ciclofosfamida, azatioprina, mofetil micofenolato, rituximab, entre otros ${ }^{16-18}$.

El pronóstico del síndrome de DRESS está determinado principalmente por el grado de compromiso hepático, siendo la hepatitis fulminante la principal causa de muerte, que llega a $10 \%$ de los $\operatorname{casos}^{1,8}$.

Considerando los escasos reportes descritos en la literatura de compromiso pulmonar en síndrome de DRESS, sumado al poco detalle que entregan al respecto las series retrospectivas más numerosas, es fundamental generar nuevos estu- dios que caractericen mejor esta manifestación clínica de este heterogéneo síndrome.

A su vez, es mandatorio considerar el síndrome de DRESS dentro del diagnóstico diferencial de los pacientes con manifestaciones cutáneas y síntomas respiratorios, asociados eventualmente a fármacos, además de evaluarlos multidisciplinariamente, de tal forma de tratarlos de forma oportuna, para así evitar complicaciones que pueden ser mortales.

\section{Referencias}

1. Husain Z, Reddy BY, Schwartz RA. DRESS syndrome: Part I. Management and therapeutics. J Am Acad Dermatol 2013; 68: 709.e1-9.

2. Choudhary S, McLeod M, Torchia D, Romanelli P. Drug Reaction with Eosinophilia and Systemic Symptoms (DRESS) Syndrome. J Clin Aesthet Dermatol 2013; 6: 31-7.

3. Bocquet H, Bagot M, Roujeau JC. Drug-induced pseudolymphoma and drug hypersensitivity syndrome (Drug Rash with Eosinophilia and Systemic Symptoms: DRESS). Semin Cutan Med Surg 1996; 15: 250-7.

4. Bessmertny O, Hatton RC, González-Peralta RP. Antiepileptic hypersensitivity syndrome in children. Ann Pharmacother 2001; 35: 533-8.

5. Fernando SL. Drug-reaction eosinophilia and systemic symptoms and drug-induced hypersensitivity syndrome. Australas J Dermatol 2014; 55: 15-23.

6. Kano Y, Ishida T, Hirahara K, Shiohara T. Visceral involvements and long-term sequelae in drug-induced hypersensitivity syndrome. Med Clin North Am 2010; 94: 743-59.

7. Cacoub P, Musette P, Descamps V, Meyer O, Speirs C, Finzi L, et al. The DRESS syndrome: a literature review. Am J Med 2011; 124: 588-97.

8. Kardaun SH, Sekula P, Valeyrie-Allanore L, Liss Y, Chu CY, Creamer D, et al. Drug reaction with eosinophilia and systemic symptoms (DRESS): an original multisystem adverse drug reaction. Results from the prospective RegiSCAR study. Br J Dermatol 2013; 169: 1071-80.

9. Ahluwalia J, Abuabara K, Perman MJ, Yan AC. Human herpesvirus 6 involvement in paediatric drug hypersensitivity syndrome. Br J Dermatol 2015; 172: 1090-5.

10. Stern RS. Clinical practice. Exanthematous drug eruptions. N Engl J Med 2012; 366: 2492-501.

11. Kano Y, Shiohara T. The variable clinical picture of drug-induced hypersensitivity syndrome/drug rash with eosinophilia and systemic symptoms in relation to the 
eliciting drug. Immunol Allergy Clin North Am 2009; 29: 481-501.

12. Yee BE, Nguyen NH, Lee D. Extensive pulmonary involvement with raltegravir induced DRESS syndrome in a postpartum woman with HIV. BMJ Case Rep 2014; 2014.

13. Augusto J-F, Sayegh J, Simon A, Croue A, Chennebault $\mathrm{J}-\mathrm{M}$, Cousin $\mathrm{M}$, et al. Extensive pulmonary involvement with raltegravir-induced DRESS syndrome in a postpartum woman with HIV. Nephrol Dial Transplant Off Publ Eur Dial Transpl Assoc - Eur Ren Assoc 2009; 24: 2940-2.

14. Bernez A, Perrinaud A, Abdallah-Lotf M, Magro P, Machet L. DRESS syndrome with severe pulmonary involvement following oral intake of a homeopathic drug. Ann Dermatol Venereol 2008; 135: 140-2.
15. Kardaun SH, Sidoroff A, Valeyrie-Allanore L, Halevy S, Davidovici BB, Mockenhaupt M, et al. Variability in the clinical pattern of cutaneous side-effects of drugs with systemic symptoms: does a DRESS syndrome really exist? Br J Dermatol 2007; 156: 609-11.

16. Descamps V, Ben Saï B, Sassolas B, Truchetet F, Avenel-Audran M, Girardin P, et al. Management of drug reaction with eosinophilia and systemic symptoms (DRESS). Ann. Dermatol. Venereol 2010; 137: 703-8.

17. Funck-Brentano E, Duong TA, Bouvresse S, Bagot M, Wolkenstein P, Roujeau JC, et al. Therapeutic management of DRESS: a retrospective study of 38 cases. J Am Acad Dermatol 2015; 72: 246-52.

18. Husain Z, Reddy BY, Schwartz RA. DRESS syndrome: Part II. Management and therapeutics. J Am Acad Dermatol 2013; 68: 709.e1-9. 